\title{
„Unser Ziel ist es, Diabetes zu heilen"
}

\author{
Novo Nordisk ist Weltmarktführer in der Diabetes-Therapie. Über die spezielle \\ Unternehmensphilosophie des dänischen Unternehmens, das einer Stiftung \\ gehört, und über die Hemmnisse für Ärzte in Deutschland, Innovationen ein- \\ zusetzen, sprach Springer Medizin mit Dr. Gundula Schneidewind und Dr. Jörn \\ Oldigs von Novo Nordisk.
}

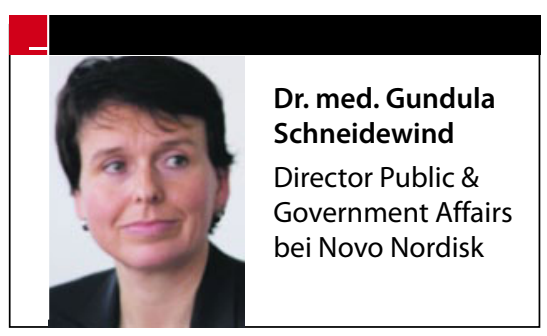

MMW: Das AMNOG ist nun mehrere Monate alt. Bewegen Sie sich inzwischen auf sicherem Terrain?

Schneidewind: Die frühe Nutzenbewertung ist eine sehr einschneidende strukturelle Veränderung, deren Folgen wir für Arzneimittelinnovationen noch nicht abschätzen können. Vor allem, wenn sie chronische Erkrankungen wie Diabetes betreffen. So werden wesentliche Surrogatparameter wie der $\mathrm{HbA}_{1 \mathrm{c}}$-Wert vom IQWiG nicht anerkannt.

MMW: Was fordert das IQWiG von Ihnen? Schneidewind: Nachweise verbesserter Morbidität und Mortalität ...

MMW: ... das kann lange dauern ...

Schneidewind: ... in der Tat. Anerkannt wird auch eine Verbesserung bei Komorbiditäten, die aber als Folge von mikro- oder makrovaskulären Schäden erst sehr spät auftreten. Beispiel: Niereninsuffizienz oder Erblindung.

MMW: Ist das überhaupt feststellbar im Rahmen von Phase-III-Studien?

Schneidewind: Nein. Die Vorgaben für diese Studien lauten: eine Blutzuckersenkung mit möglichst wenig Nebenwirkungen zu erreichen. Denn desto besser kann der Patient geführt werden, und desto besser kann er seinen Blutzuckerhaushalt in der Norm halten und Folgeerkrankungen vermeiden.

MMW: Die Entwicklung Ihres Portfolios ist langfristig geplant. Wie steht es bei Degludec? Oldigs: Die Phase-III-Studien sind abgeschlossen und wir planen, die Zulassung für dieses ultralangwirksame Basalinsulin dieses Jahr einzureichen.

MMW: Welche Eigenschaften hat dieses Insulin?

Oldigs: Es geht darum, eine bestimmte $\mathrm{HbA}_{1 \mathrm{c}}$-Senkung zu erreichen und vor allem nächtliche Hypoglykämien zu vermeiden. Das vergleichen wir mit dem Marktführer, dem Insulin-Glargin. Und hier zeigen die Studien, dass die Häufigkeit von Hypoglykämien insgesamt um 16\%, die der nächtlichen sogar um $32 \%$ gesenkt wird.

Schneidewind: Außerdem kann dieses Insulin unabhängig von der Tageszeit genommen werden, weil das Blutzuckerprofil sehr gleichmäßig bleibt.

MMW: Das wäre ja vorteilhaft für die Compliance. Würde dies das IQWiG als Kriterium berücksichtigen?

Schneidewind: Überhaupt nicht. Auch wenn ein Arzneimittel statt täglich nur einmal wöchentlich gegeben werden müsste das IQWiG sähe darin keinen Zusatznutzen.

MMW: Eigentlich müsste es ja einen Aufstand der Ärzte geben.

Schneidewind: Ich glaube, vielen Ärzten ist gar nicht bewusst, was da geschieht.

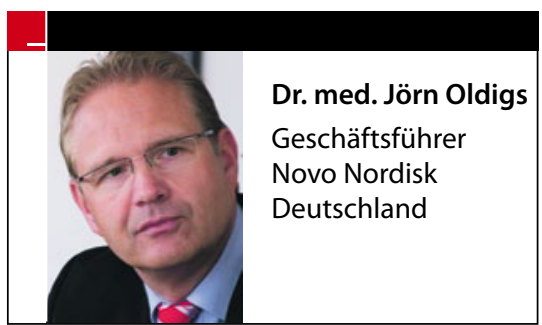

Oldigs: Deutschland ist das einzige Land weltweit, wo moderne Insulintherapie zu den gleichen Kosten angeboten wird wie 30 Jahre alte Humaninsuline. Trotzdem haben die Insulinanaloga hier im Vergleich die geringsten Marktanteile. Dabei haben wir für NovoRapid ${ }^{\circledR}$ und NovoMix ${ }^{\circledast} 30$ bereits seit 2006 Rabatte. Für Levemir ${ }^{\circledR}$ haben wir mit fast allen Krankenkassen letztes Jahr Versorgungswahlverträge abgeschlossen, die die vom GBA geforderte Kostengleichheit garantieren. Trotzdem werden immer noch überwiegend Humaninsuline eingesetzt.

MMW: Was verbirgt sich hinter „Changing Diabetes"?

Oldigs: Es ist ein Langfrist-Programm, das sich aus der Geschichte des Unternehmens und einer besonderen Betroffenheit erklärt. Die Frau des Gründers, Marie Krogh, hatte Diabetes; das war der Grund, nach einer Therapie zu suchen. Novo Nordisk ist eine Stiftung, die das Ziel hat, Diabetes zu heilen.

MMW: Ist das nicht auch ein Kampf gegen Windmühlen?

Oldigs: Gerade die steigende Zahl von Diabetikern spornt uns an. Fatalismus wäre falsch, und davon versuchen wir auch Politik und Gesellschaft zu überzeugen. Wenn man nicht präventiv agiert, dann ist die Welle, die auf uns zurollt, so groß, dass ein Viertel der Gesundheitsausgaben allein durch Diabetes verursacht wird.

- Das Interview führten Wolfgang van den Bergh, Wolfgang Geissel und Helmut Laschet. 